\title{
An establishment of exo-erythrocytic stage of malaria parasite for ex-vivo study of sporozoiticidals
}

Sharma Supriya, Kumar Navin

Epidemiology \& Clinical Research Division National Institute of Malaria Research (ICMR)

Eur J Basic Med Sci 2013;3(1): 22-23

Received: 10-09-2012

Accepted: 10-12-2012

Correspondence (Yazıșma Adresi):

Supriya Sharma

Epidemiology \& Clinical Research Division National Institute of Malaria Research (ICMR) Sector 8, Dwarka, New Delhi -

110077, India

Phone number - 09990299979

E-mail address - supsmicro@gmail.com

\section{Dear Editor,}

Despite, since 1880s plasmodium blood stages have been recognized, our knowledge of liver stage parasite is still six decade old. Even after a long time of its discovery, the exo-erythrocytic forms of the parasite have remained adamant to reveal their biological secrets. Till date, our understanding of liver stage of parasite is in vague. Infection in human is initiated when plasmodium sporozoite enter through the bite of female Anopheles mosquito during their obligatory blood meal (1). Under the skin of host an average of $15-123$ sporozoites have been reported to be deposited where they infect hepatocytes and begin to develop into merozoites (2). Depending on the species of plasmodium, thousands of merozoites per invading sporozoite between 2 and 16 days are released in blood stream for erythrocytic infection. Although the obligatory step of sporozoite establishment and full development inside is symptomatically silent, it gives rise to thousand of merozoites in the hepatocyte.

All our efforts related to eradication of malaria to date are towards the erythrocytic stage of the parasite as a consequence very few drugs are available for exo-erythrocytic form of parasite. An appealing target for the antimalarial vaccine or prophylactic drug as they would function before the onset of pathology is the hepatic stage (3). In order to address the unmet need of drug target for hepatocyte stage parasite an ex-vivo system should be establish so as to discover a sporozoiticidal drug for prophylactic treatment of malaria. The hepatoma cell line constitutes an invaluable and widely used tool to study the aspect of hepatic infection by plasmodium. Till date it has been proven to be ideal for rodent parasite. These days the advancement is towards the human cell line which could support the growth of human parasite Plasmodium falciparum (4). To some extent the cell line HC-04 has proven its worth but still some fundamental question are needed to be answered. These present advance lays a ground for a standardization of an ex-vivo system through which we gain an acceleration in the progress of malaria elimination research. 


\section{Acknowledgement:}

The author is thankful to Indian Council of Medical Research (ICMR), for the financial support and Dr. C. R. Pillai, Emeritus Scientist, Dr. Anup Anvikar, Scientist 'D' of National Institute of Malaria Research, New Delhi.

\section{KAYNAKLAR}

1. Derbyshire ER, Mota MM, Clardy J. The Next Opportunity in Anti-Malaria Drug Discovery: The Liver Stage. PloS Pathog 2011; 7:1-5.
2. Derbyshire ER, Prudencio M, Mota MM, Clardy J. Liverstage malaria parasites vulnerable to diverse chemical scaffolds. PNAS 2012; 109: 8511-6.

3. Prudencio M, Rodriguez A, Mota MM. The silent path to thousands of merozoites: the plasmodium liver stage. Nature Reviews 2006; 4:849-56.

4. Kappe SHI, Duffy P. Editorial malaria liver stage culture: In Vitro Veritas? Am J. Trop. Med Hyg 2006; 74(5): 706-7. 